\title{
Concomitant Medication Dose Unit
}

National Cancer Institute

\section{Source}

National Cancer Institute. Concomitant Medication Dose Unit. NCI Thesaurus. Code C83034.

The unit of measure for the concomitant medication dose. 